\title{
Efficient negative ion production in rf plasmas using a mesh grid bias method ${ }^{\text {a) }}$
}

\author{
Junichi Okada, Yuichi Nakao, Yasushi Tauchi, and Osamu Fukumasa ${ }^{\text {b) }}$ \\ Graduate School of Science and Engineering, Yamaguchi University, Tokiwadai 2-16-1, Ube 755-8611, Japan
}

(Received 27 August 2007; presented 29 August 2007; accepted 8 October 2007; published online 15 January 2008)

Using a grid bias method for plasma parameter control, volume production of hydrogen negative ions $\mathrm{H}^{-}$is studied in pure hydrogen rf plasmas. Relationship between the extracted $\mathrm{H}^{-}$ion currents and plasma parameters is discussed. It is confirmed that both high and low electron temperature $T_{e}$ plasmas are produced in the separated regions when the grid is negatively biased. In addition, with changing grid potential $V_{g}$, values of $n_{e}$ increase while $T_{e}$ decrease in their values. The negative ion production depends strongly on the grid potential and related plasma conditions. () 2008 American Institute of Physics. [DOI: 10.1063/1.2805372]

\section{INTRODUCTION}

Development of negative ion sources for neutral beam injection (NBI) system is required for large experimental fusion devices such as the ITER. In the present negative ion sources, source plasma is generated by dc arc discharge, so the lifetime of the ion source is limited to several hundred hours due to erosion and fatigue of the cathode filaments. Thus a long-lifetime ion source is required for future NBI systems. Microwave-discharge ion sources ${ }^{1}$ and $\mathrm{rf}$-driven ion sources $^{2,3}$ are promising as long-lifetime ion sources because they have no filaments.

In pure hydrogen $\left(\mathrm{H}_{2}\right)$ plasmas, most of the $\mathrm{H}^{-}$ions are generated by the dissociative attachment of slow plasma electrons (electron temperature $T_{e} \sim 1 \mathrm{eV}$ ) to highly vibrationally excited hydrogen molecules $\mathrm{H}_{2}\left(v^{\prime \prime}\right) .{ }^{4}$ These molecules are mainly produced by collisional excitation of fast electrons with energies of $15-20 \mathrm{eV}$. Therefore, spatial control of electron energy distribution (i.e., $T_{e}$ ) is necessary., With the use of a magnetic filter, the electron energy distribution function in dc plasmas is well controlled for $\mathrm{H}^{-}$formation. Unfortunately, in rf discharge plasmas, plasma parameter control with magnetic filter is not so effective as one in dc plasmas. ${ }^{1,2}$ Therefore, using a grid bias method ${ }^{7,8}$ for plasma parameter control, volume production of hydrogen negative ions $\mathrm{H}^{-}$is studied in pure hydrogen rf plasmas.

The purpose of the present study are as follows: one is to investigate the possibility of controlling plasma parameters, in particular $T_{e}$, with grid bias method in rf-driven plasmas; and the other is to realize negative ion production in rf plasmas and to discuss the difference in $T_{e}$ control and $\mathrm{H}^{-}$production between the mesh grid bias method and the magnetic filter method.

\footnotetext{
a) Contributed paper, published as part of the Proceedings of the 12th International Conference on Ion Sources, Jeju, Korea, August 2007.

${ }^{b)}$ Author to whom correspondence should be addressed. FAX: +81-836-859401. Electronic mail: fukumasa@plasma.eee.yamaguchi-u.ac.jp.
}

\section{EXPERIMENTAL SETUP AND PROCEDURE}

Figure 1 shows a schematic diagram of rf negative ion source. The source chamber $(21 \mathrm{~cm}$ in diameter) made of stainless steel is divided by a mesh grid (MG) into two parts, i.e., a source region and an extraction region. The rf power $(13.56 \mathrm{MHz})$ is supplied to a stainless circular disk antenna with $12 \mathrm{~cm}$ diameter. In the experiment, we use hydrogen $\left(\mathrm{H}_{2}\right)$ gases under a pressure of 3 mTorr. rf power is varied from 100 to $300 \mathrm{~W}$. To produce both high and low electron temperature plasmas separately in the chamber, a grid $20 \mathrm{~cm}$ in diameter is placed $18.5 \mathrm{~cm}$ away from the rf antenna. In the experiment, three different meshes are used, i.e., No. 1 (7 mesh/in.), No. 2 (30 mesh/in.), and No. 3 (50 mesh/in.). Details are shown in Table I. Compared with the usual magnetically filtered multicusp ion source, in Fig. 1, the magnetic filter (MF) flange (i.e., the rod-type filter) is set instead of the MG flange. The present MF is composed of four rods where diameter of the rod is $10 \mathrm{~mm}$ and the distance between two rods is $54 \mathrm{~mm}$. In the present experiment, magnetic field intensities of the MF are 60 and $100 \mathrm{G}$. The negative ion currents are directly detected by a magnetic deflection-type ion analyzer. The plasma grid has a single hole (10 $\mathrm{mm}$ in diameter) through which negative ions were extracted from the ion source. Plasma parameters (electron density $n_{e}$, electron temperature $T_{e}$, plasma space potential $V_{s}$, and floating potential $V_{f}$ ) are measured by three Lang-

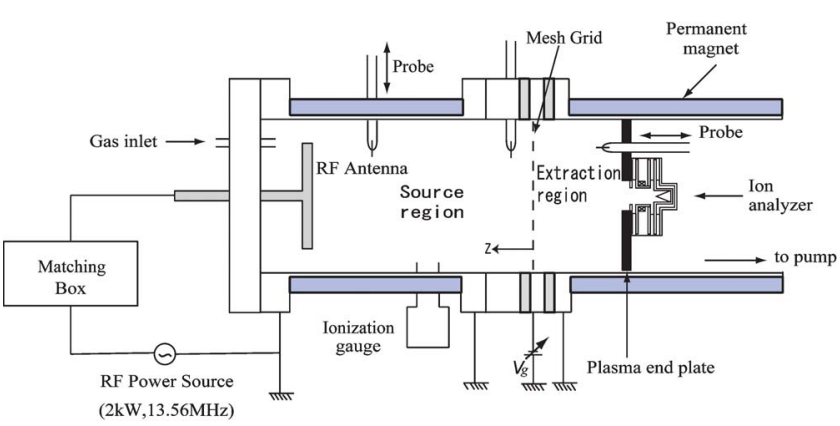

FIG. 1. (Color online) Schematic diagram of the experimental apparatus. 
TABLE I. Dimensions of three meshes used in the present experiment.

\begin{tabular}{|c|c|c|c|c|}
\hline Mesh & $\begin{array}{l}\text { Mesh size } \\
\text { (mesh/in.) }\end{array}$ & $\begin{array}{l}\text { Diameter of wire } \\
(\mathrm{mm})\end{array}$ & $\begin{array}{l}\text { Distance between } \\
\text { two wires } \\
(\mathrm{mm})\end{array}$ & $\begin{array}{c}\text { Geometric } \\
\text { transmittance }(\%)\end{array}$ \\
\hline No. 1 & 7 & 1.03 & 2.36 & 48.5 \\
\hline No. 2 & 30 & 0.25 & 0.597 & 49.7 \\
\hline No. 3 & 50 & 0.05 & 0.458 & 81.3 \\
\hline
\end{tabular}

muir probes. The one is movable along the axial direction from the mesh grid in the extraction region. The others are set at $z=3.7 \mathrm{~cm}$ and $z=11 \mathrm{~cm}$ in the source region.

\section{EXPERIMENTAL RESULTS AND DISCUSSION}

\section{A. Plasma production and control}

The aim of the present work is production and control of rf plasmas to enhance and to optimize $\mathrm{H}^{-}$volume production in rf plasmas.

Figure 2 shows axial distributions of plasma parameters (i.e., $n_{e}$ and $T_{e}$ ) controlled by the MF. In the extraction region, $T_{e} \simeq 4 \mathrm{eV}$. The value of $T_{e}$ is high for negative ion production. In addition, $n_{e}$ decrease drastically in the extraction region. Although $T_{e}$ is decreased by increasing the field intensity of the MF, at the same time, $n_{e}$ is also decreased more drastically. On negative ion volume production, a desired condition for plasma parameters is as follows: $T_{e}$ in the extraction region should be reduced below $1 \mathrm{eV}$ with $n_{e}$ keeping higher. Therefore, in rf plasmas, plasma parameter control with using the MF is not so effective.

Similar measurements have been made with using a mesh grid bias method. Figure 3 shows axial distributions of plasma parameters for three different MGs. High energy electrons pass the mesh (set at $z=0 \mathrm{~cm}$ ) and enter into the extraction region. As is shown clearly, $n_{e}$ increases in its value with $z$ and reaches the maximum value and then decreases while $T_{e}$ decreases in its value and keeps nearly equal to or lower than $1 \mathrm{eV}$. This is suitable condition for negative ion volume production. The mechanism is explained as follows: In the extraction region, the neutral particles are ionized by higher energy electrons flowing from the source region through the grid. The electrons produced in the extraction region cannot be accelerated by the external electric fields, because no additional heating power is fed
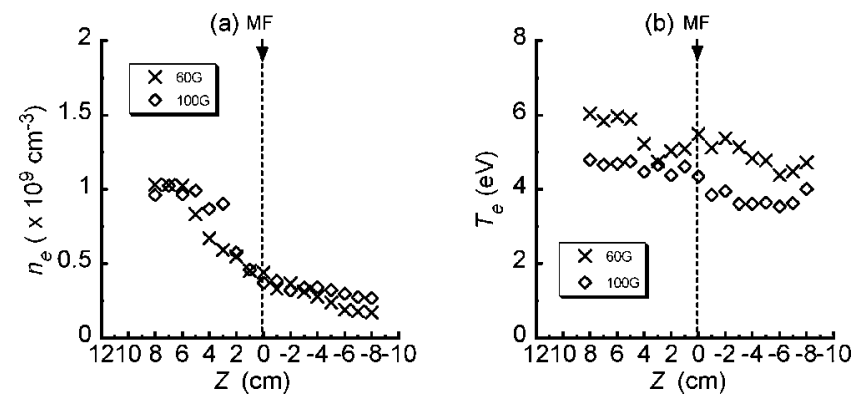

FIG. 2. Axial distributions of plasma parameters: Electron density $n_{e}$ (a) and electron temperature $T_{e}$ (b) for two different MF fields. The end plate is set at $z_{\text {end }}=-10.5 \mathrm{~cm}$. Experimental conditions: $P_{\mathrm{rf}}=200 \mathrm{~W}$ and $p\left(\mathrm{H}_{2}\right)=3$ mTorr.
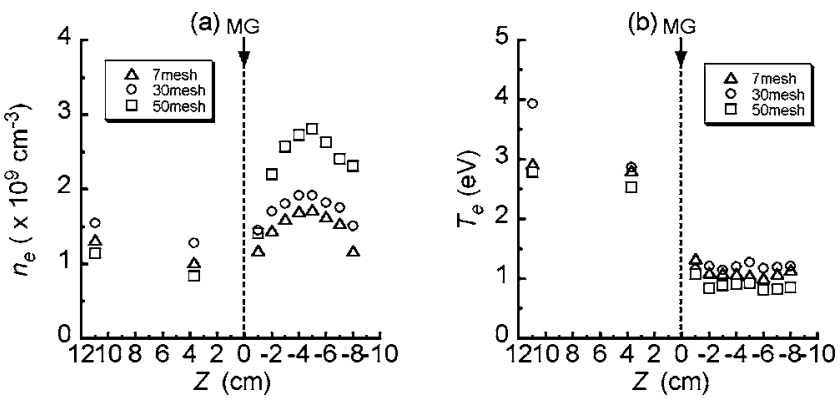

FIG. 3. Axial distributions of plasma parameters: (a) $n_{e}$ and (b) $T_{e}$ for three different mesh grids. The end plate is set at $z_{\text {end }}=-10.5 \mathrm{~cm}$. Experimental conditions: $P_{\mathrm{rf}}=200 \mathrm{~W}, p\left(\mathrm{H}_{2}\right)=3 \mathrm{mT}$ Torr, and grid biasing voltage $V_{g}=-50 \mathrm{~V}$

into this region. Therefore, low energy electrons are generated in the extraction region. According to the results shown in Figs. 2 and 3, the grid bias method is more suitable to optimize plasma conditions for negative ion, compared with the magnetic filter method.

Figure 4 shows the dependence of plasma parameters on grid bias voltage for three different MGs in the extraction region. With changing $V_{g}$, values of $n_{e}$ increase while $T_{e}$ decrease in their value. There appears a decrease in $T_{e}$ with a decrease in grid biasing voltage $V_{g}$. We also measure the $T_{e}$ at $z=3.7 \mathrm{~cm}$ in the source region and obtain $T_{e} \simeq 3 \mathrm{eV}$, which is almost independent of $V_{g}$. Therefore, $T_{e}$ in the extraction region has a lower value than that in the source region. Behaviors of plasma parameters are nearly the same as the ones in dc plasmas. ${ }^{10,11}$

Grid bias method is the method to control electron transport electrostatically and is affected by the mesh size. ${ }^{7}$ Then, with using three different MGs, the effect of mesh size is also tested. We estimate the Debye length $\left(\lambda_{D}\right)$ from the typical plasma parameters (i.e., $n_{e} \simeq 1 \times 10^{9} \mathrm{~cm}^{-3}$ and $T_{e} \simeq 3.0 \mathrm{eV}$ ) at $z=3.7 \mathrm{~cm}$ in the source region and obtain that $\lambda_{D} \simeq 0.41 \mathrm{~mm}$. When we suppose that the sheath length is about several times of $\lambda_{D}$, distances between two wires of all meshes are within the sheath length. In the extraction region, $n_{e}$ with No. 3 mesh is higher than other two meshes (i.e., No. 1 and No. 2). This is caused mainly by the difference of transmittance. It is easy for electrons cross the MG and enter into the extraction region as No. 3 mesh has high transmittance.

We have confirmed numerically that extraction probabil-
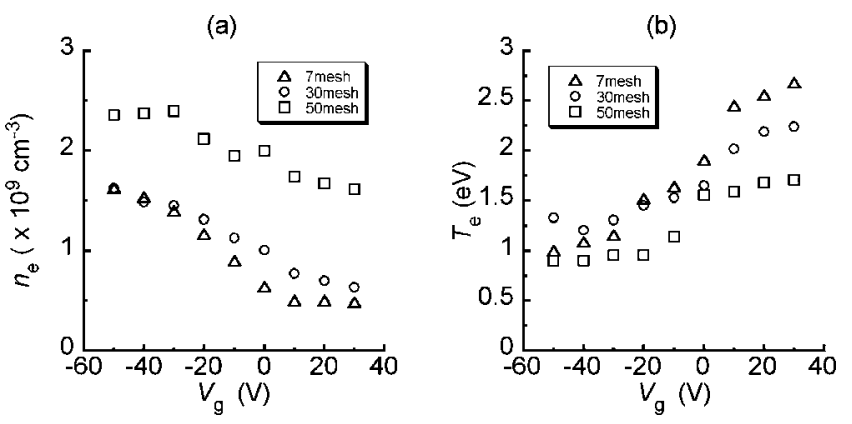

FIG. 4. (a) $n_{e}$ and (b) $T_{e}$ vs grid potential $V_{g}$ at $z=-5 \mathrm{~cm}$ in the extraction region for three different mesh grids. The end plate is set at $z_{\text {end }}=-10.5 \mathrm{~cm}$. Experimental conditions: $P_{\mathrm{rf}}=200 \mathrm{~W}$ and $p\left(\mathrm{H}_{2}\right)=3 \mathrm{mTorr}$. 


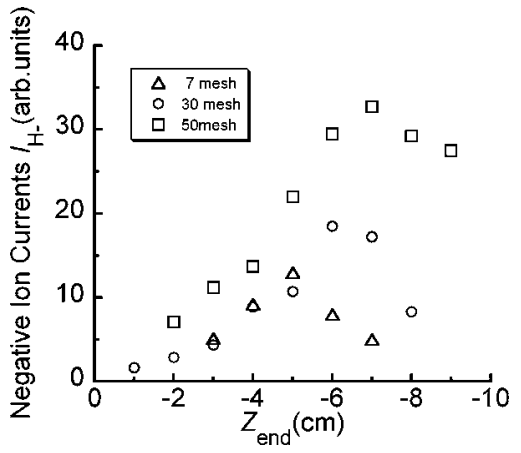

FIG. 5. Extracted negative ion currents as a function of the end plate position for three different mesh grids. Experimental conditions: $P_{\mathrm{rf}}=200 \mathrm{~W}$, $p\left(\mathrm{H}_{2}\right)=3$ mTorr, grid biasing voltage $V_{g}=-50 \mathrm{~V}$, and $V_{\mathrm{ex}}=1 \mathrm{kV}$.

ity of negative ions depends strongly on upstream distance from the extraction grid, ${ }^{12}$ i.e., the plasma end plate in the present case. At any rate, to increase the extraction of negative ion currents, production of negative ions near the plasma end plate should be enhanced by optimizing plasma conditions.

\section{B. Negative ion production}

We discuss $\mathrm{H}^{-}$ion production in the present rf plasmas. According to the results shown in Fig. 3, plasma parameters in the extraction region depend on the position of the end plate, and then production of negative ions. Figure 5 shows the effect of end plate position $z_{\text {end }}$ on extracted negative ion currents $I_{\mathrm{H}^{-}}$(i.e., $\mathrm{H}^{-}$ion production). At first, $I_{\mathrm{H}^{-}}$increases with $z_{\text {end }}$, reaches maximum at $z_{\text {end }}=-5$ to $-7 \mathrm{~cm}$, and then decreases. The changes in $I_{\mathrm{H}^{-}}$are caused by the plasma parameters in the extraction region. Plasma production and then $\mathrm{H}^{-}$ion production also depended on hydrogen gas pressure $p\left(\mathrm{H}_{2}\right)$.

Figure 6 shows the rf power dependence of $I_{\mathrm{H}^{-}}$. According to the results shown in Fig. 5 and pressure dependence of

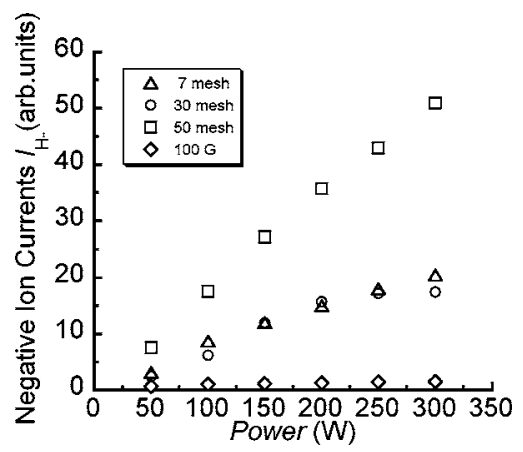

FIG. 6. Extracted negative ion currents as a function of discharge power for three different mesh grids and the magnetic filter method. Experimental conditions: $p\left(\mathrm{H}_{2}\right)=3$ mTorr, grid biasing voltage $V_{g}=-50 \mathrm{~V}$, and $z_{\text {end }}=-5 \mathrm{~cm} \quad(7 \mathrm{mesh} /$ in. $), \quad z_{\text {end }}=-6 \mathrm{~cm} \quad(30 \mathrm{mesh} / \mathrm{in}),. \quad z_{\text {end }}=-7 \mathrm{~cm}$ (50 mesh/in.) for grid bias method. $p\left(\mathrm{H}_{2}\right)=2 \mathrm{mTorr}, z_{\text {end }}=-4 \mathrm{~cm}$ for the magnetic filter method, and $V_{\mathrm{ex}}=1 \mathrm{kV}$. the $\mathrm{H}^{-}$production, pressure and $z_{\text {end }}$ are optimized. In addition, rf power dependence of $I_{\mathrm{H}^{-}}$with magnetic filter method is shown in Fig. 6 for comparison. With increasing power, $I_{\mathrm{H}^{-}}$increases linearly. $I_{\mathrm{H}^{-}}$in the grid bias method is much higher than that in the magnetic filter method. It is clear from plasma parameter. Within the present experimental conditions, plasma production and control in the extraction region well done for negative ion production when No. 3 mesh is set due to high transmittance.

\section{SUMMARY}

Control of plasma parameters and enhancement of extracted $\mathrm{H}^{-}$ion currents in $\mathrm{rf}$ plasmas are studied by using the magnetic filter method and the mesh grid bias method. With using the magnetic filter method, electron temperature in the extraction region is not well controlled for $\mathrm{H}^{-}$ion formation. However using the mesh grid bias method, we have confirmed that both high and low electron temperature plasmas are produced in the separated regions in the chamber, respectively, when grid potential $V_{g}$ is negative. Extracted negative ion currents $I_{\mathrm{H}^{-}}$depend on some experimental conditions and $I_{\mathrm{H}^{-}}$in the grid bias method is much higher than that in the magnetic filter method. As, in the future, rf negative ion source is required for the NBI systems; the mesh grid bias method is quite useful to control and enhance negative ion volume production in $\mathrm{rf}$ plasmas.

\section{ACKNOWLEDGMENTS}

The authors would like to thank Professor Emeritus Noriyoshi Sato, Tohoku University, for his interest and encouragement to this work. They also thank Y. Tauchi for his assistance. A part of this work was supported by the Grantin-Aid for Scientific Research from Japan Society for the Promotion of Science.

${ }^{1}$ O. Fukumasa and M. Matsumori, Rev. Sci. Instrum. 71, 935 (2000).

${ }^{2}$ T. Takanashi, Y. Takeiri, O. Kaneko, Y. Oka, K. Tsumori, and T. Kuroda, Rev. Sci. Instrum. 67, 1024 (1996).

${ }^{3}$ E. Speth, M. D. Falter, P. Franzen, U. Fantz, M. Bandyopadhyay, S. Christ, A. Enchena, M. Fröschle, D. Holtum, B. Heinemann, W. Kraus, A. Lorenz, Ch. Martens, P. McNeely, S. Obermayer, R. Riedl, R. Juss, A. Tanga, R. Wilhelm, and D. Wünderlich, Nucl. Fusion 46, S220 (2006).

${ }^{4}$ O. Fukumasa, J. Phys. D 22, 1668 (1989).

${ }^{5}$ O. Fukumasa, S. Mori, N. Nakada, Y. Tauchi, M. Hamabe, K. Tsumori, and Y. Takeiri, Contrib. Plasma Phys. 44, 516 (2004).

${ }^{6}$ O. Fukumasa and S. Mori, Nucl. Fusion 26, S287 (2006).

${ }^{7}$ K. Kato, S. Iizuka, and N. Sato, Appl. Phys. Lett. 65, 816 (1994).

${ }^{8}$ S. Iizuka, K. Kato, A. Takahashi, K. Nakagomi, and N. Sato, Jpn. J. Appl. Phys., Part 1 36, 4551 (1997)

${ }^{9}$ Y. Nakao, D. Ito, J. Ono, Y. Tauchi, and O. Fukumasa, Proceedings of the Sixth International Conference on Reactive Plasmas and 23rd Symposium on Plasma Processing, Matsushima/Sendai, 2006 (unpublished), p. 185.

${ }^{10}$ O. Fukumasa, D. Ito, and Y. Jyobira, 11th International Symposium Production and Neutralization of Negative Ions and Beams, 13-15 September 2006 (unpublished).

${ }^{11}$ Y. Jyobira, D. Ito, and O. Fukumasa, 12th International Conference on Ion Sources, 26-31 August 2007 (unpublished).

${ }^{12}$ O. Fukumasa and R. Nishida, Nucl. Fusion 46, S220 (2006). 